\title{
Les techniques du corps chez les Mongols : une application de la notion maussienne
}

Gaëlle Lacaze

\section{(2) OpenEdition}

\section{Journals}

Édition électronique

URL : https://journals.openedition.org/tc/114

DOI : $10.4000 /$ tc. 114

ISSN : 1952-420X

Éditeur

Éditions de l'EHESS

\section{Édition imprimée}

Date de publication : 1 avril 2004

Pagination : 111-130

ISSN : 0248-6016

\section{Référence électronique}

Gaëlle Lacaze, «Les techniques du corps chez les Mongols : une application de la notion

maussienne », Techniques \& Culture [En ligne], 42 | 2004, mis en ligne le 06 novembre 2007, consulté le 29 septembre 2022. URL : http://journals.openedition.org/tc/114 ; DOI : https://doi.org/10.4000/tc

114

Ce document a été généré automatiquement le 29 septembre 2022

Tous droits réservés 


\title{
Les techniques du corps chez les Mongols : une application de la notion maussienne
}

\author{
Gaëlle Lacaze
}

1 Marcel Mauss présente la «Notion de technique du corps» le 17 mai 1934 lors d'un séminaire de la Société de Psychologie (1989 : 365-386). Il y définit ces «ritualités en acte » en tant qu' « ouvrage de la raison pratique collective et individuelle» (1989: 372). Il posait ainsi les fondements d'une réflexion sur la gestualité, mais sans la développer, car il ne l'a pas appliquée à l'étude d'une société particulière. Cet article propose de le faire pour le cas des Mongols. Après une présentation des populations ${ }^{1}$, j'analyserai la notion de corps et le vocabulaire qui s'y rattache. Puis j'aborderai le processus d'éducation, de «dressage» des enfants, et enfin les techniques du corps de la personne adulte. Ce travail, qui révèle un rapport étroit entre les représentations du corps, de la personne et de l'espace, invite à une relecture de la notion maussienne.

Contexte, méthode et définitions

2 Aujourd'hui, les Mongols forment un groupe hétérogène constitué de plusieurs sociétés qui ont en commun une langue et un fonds culturel basé sur le pastoralisme nomade comme sur la forme de chamanisme qui lui est associée. Les populations sont localisées en Mongolie, en Russie et dans le nord de la Chine, en République Autonome de Mongolie-Intérieure (RAMI), et dans le Turkestan.

Les peuples mongols

3 Les Mongols ont deux systèmes de croyances: le chamanisme et le bouddhisme tibétain, plus particulièrement la branche réformée des «Bonnets jaunes » (Gelug-pa, tb. dge lugs-pa). Le bouddhisme fut introduit à plusieurs reprises depuis le Moyen Âge chez les Mongols de tradition chamanique. À la fin du XVIe siècle, ils se convertissent massivement au bouddhisme "jaune», qui restera la religion officielle de Mongolie jusqu'en 1924. Entre 1911 et 1921, le chef de l'église bouddhiste mongole, le Jebstundamba hutugtu ou Bogdo gegen, occupe les fonctions de chef d'État. De nos jours, le bouddhisme "jaune " est considéré comme la "religion traditionnelle mongole», 
tandis que le chamanisme est la "croyance ancestrale des Mongols». En outre, le bouddhisme qui pénètre chez les Mongols est empreint d'anciennes croyances populaires tibétaines (Even 1998 : 149-196). Il trouve des parallèles dans le chamanisme des sociétés d'élevage, ce qui facilite la bouddhisation des Mongols, mais aussi l'adaptation et la survie de ce système rituel.

4 Au XXe siècle, à la suite de la Révolution populaire de 1921, l'administration mongole développe une idéologie socialiste et athéiste, d'obédience soviétique. Le bouddhisme constitue alors la cible privilégiée de l'épuration religieuse. L'administration socialiste a parfois revivifié des représentations chamaniques pour l'éradiquer. Les processus d'acculturation entraînent souvent des contradictions et des décalages qui brouillent l'analyse. Chez les Mongols, des représentations du corps liées aux conceptions chamaniques sont repérables, parfois sous une forme bouddhisée.

Les techniques du corps

5 Le corps n'est pas un objet facilement pensé par les informateurs. Il est d'abord mis en actes : le fait même de produire un discours à son sujet implique sa mise en action. Autrement dit, les discours sur les pratiques, les rituels ou les techniques en la matière sont en eux-mêmes le produit d'une pratique, d'un rituel et imposent l'emploi d'une technique liée au corps : la maîtrise du langage.

6 Les techniques du corps et de soi sont incorporées par le sujet. Elles relèvent de mécanismes psychomoteurs de "dressage » et d'éducation devenant «inconscients ». Elles sont donc considérées comme «naturelles » et biologiques. Ainsi selon Mauss, la technique du corps est un « acte traditionnel efficace, senti par l'auteur comme un acte d'ordre mécanique, physique ou physico-chimique et [...] poursuivi dans ce but» (1989 : 372). La description qui en est faite par ceux qui les réalisent reflète un idéal de comportement qui ne correspond pas toujours aux faits observés sur le terrain. Les décalages entre le discours et la pratique révèlent des stratégies identitaires, sociales et individuelles et des tensions entre les différents systèmes de représentation.

7 La notion maussienne semble particulièrement appropriée à l'étude de peuples de tradition pastorale nomade. Selon lui, le «dressage » est un ensemble de "procédés appliqués aux animaux que les humains s'appliquent à eux-mêmes et à leurs enfants » (1989 : 375). Cette idée de dressage est importante chez les Mongols, car ils font souvent référence au processus de domestication de l'animal. L'assimilation des techniques du corps humain à celles du corps animal y est récurrente. Elle reflète plusieurs stratégies sociales. C'est une volonté de maîtrise lorsqu'il s'agit d'une référence au processus de domestication de l'animal. En revanche, l'assimilation de l'humain à un animal sauvage renvoie à l'expression d'une marginalité sociale (alcoolique) ou à la sphère du rituel (chamanique), elle l'exclut du champ d'expression de la "normalité ». C'est par le dressage que se développe "l'habilis", principe où se mêlent l'adresse, la présence d'esprit et l'habitude. Selon Mauss, « le dressage, comme le montage d'une machine, est la recherche, l'acquisition d'un rendement [...] humain » (1989: 375). On distinguera donc des techniques du corps à fort « rendement» et d'autres moins « rentables». La recherche d'une efficacité des techniques de dressage mises en place pendant l'enfance est en effet repérable dans les représentations de ces techniques à l'âge adulte : elles servent de support privilégié à l'expression individuelle et sociale. Elles constituent donc un objet exemplaire d'analyse. Le dressage est intense pendant l'enfance, mais nous verrons qu'il ne s'y limite pas. 
8 La notion de techniques du corps est classique en sciences humaines; elle est souvent utilisée mais rarement dans le sens où Mauss l'a définie. Celui-ci considère les techniques du corps dans leur pluralité. L'application concrète de la méthode maussienne à la société mongole débouche sur une forme "d'encyclopédie gestuelle ", à plusieurs niveaux révélatrice. Mauss insiste sur l'imbrication des niveaux de détermination biologique, psychologique et sociologique, qui impliquent «l'homme total » $(1989: 369)$. Il tente de mettre en évidence la part respective de ces trois niveaux qui «vont ensemble», et affirme la domination du social. Par exemple, dans l'art d'utiliser le corps, les processus $d^{\prime}$ '«imitation prestigieuse»dominent. Si dans l'«imitation » se situe «tout l'élément biologique et psychologique ", «tout l'élément social » se trouve quant à lui dans la notion de " prestige » (1989:369). Les techniques $\mathrm{du}$ corps varient «non pas seulement avec les individus et leurs imitations », mais " surtout avec les sociétés, les éducations, les convenances et les modes, les prestiges » (1989: 368). Il y a "peu de création de positions de principe", mais plutôt des adaptations psychologiques individuelles, "généralement commandées par l'éducation, et au moins par les circonstances de la vie en commun, du contact ». Les techniques du corps sont donc une « série d'actes physio-psycho-sociologiques montée chez l'individu par et pour l'autorité sociale » $(1989: 384)$. Le niveau social, la société, ou la vie sociale déterminent les « engrenages » psychologiques.

9 L'étude des techniques de dressage mongoles met en évidence l'importance de la notion d'imitation, qui consiste surtout dans l'adoption d'un modèle caractéristique de son statut, social et individuel. En effet, imiter des comportements propres à une catégorie supérieure ne procure aucun prestige. En principe, il faut se comporter comme un enfant pendant l'enfance et ne plus le faire à l'adolescence ou à l'âge adulte. Les enfants tirent leur prestige de la maîtrise des techniques du corps dévolues à leur âge, leur sexe et leur statut. On remarque que savoir jouer aux « jeux de son âge » et avec des enfants de statut équivalent est une source de prestige. En revanche, jouer à des jeux qui ne sont pas «de son âge » suscite le dénigrement. S'ils imitent leurs aînés, les enfants le font dans une volonté contestataire en présence d'adultes ou sous la forme de jeux apportant le prestige $\mathrm{du}$ « bon clown » au sein du groupe d'enfants.

10 "L'imitation prestigieuse » peut jouer un rôle déterminant dans les techniques du corps de l'adulte. Elle renvoie à une relation inégalitaire entre catégories socioéconomiques. Ainsi le clivage entre villes et campagnes est-il important: un éleveur récemment arrivé en ville peut se reconnaître à ses vêtements, mais aussi à sa démarche. Même s'ils tirent un prestige dans l'adoption de techniques du corps caractéristiques d'une catégorie supérieure à la leur, les Mongols adultes préfèrent s'en tenir aux attitudes représentatives de leur statut. Ils n'apprécient guère qu'on se « donne un air ».

11 Les techniques de dressage de l'enfance impliquent donc une dimension "d'imitation catégorielle » au sein des classes sociales. L'apprentissage s'effectue au sein de groupes relativement exclusifs en termes d'âge et de sexe. Il est marqué par le prestige procédant de la maîtrise progressive d'une technique du corps selon le statut. À l'âge adulte, la promiscuité entre personnes de catégories différentes est grande, mais la beauté des femmes, par exemple, se caractérise, entre autres, par des attitudes « comme il faut». Une fois parfaitement acquise, une technique est incorporée : elle semble mise «naturellement » en œuvre, et un nouveau processus de dressage peut commencer. 
Le corps mongol

12 Pour suivre Mauss, qui préconise d'aller du concret vers l'abstrait, il faut d'abord définir la notion de "corps » (bije) chez les Mongols. Le corps est à la fois considéré comme support de l'âme, représentant la personne, et comme réceptacle de force vitale.

13 Le terme bije peut donc prendre plusieurs sens selon les contextes. Il réfère à la santé dans l'expression quotidienne bije sain uu? (lit. «le corps, ça va?»). D’autres expressions réfèrent au corps dans le sens de "personne »: en langage soutenu, la forme possessive et réfléchie de bije (minij bije ou bijee) signifie «moi, je». Dans l'expression bijegüj (lit. « sans corps »), se trouvent à la fois l'aspect « immatériel » des esprits et la valeur négative du "corps malingre », malade ou imparfait. Cet usage privatif souligne la valorisation du corps en tant qu'entité physique achevée; de même, il place les humains en opposition avec les entités du monde surnaturel, les êtres « sans-corps».

En outre, juxtaposé à un nom, le terme bije peut exprimer la matérialité d'une chose. L'expression bouriate " corps-argent » (bije-möngö) désigne ainsi des espèces sonnantes et trébuchantes. Associé à d'autres mots, bije permet de désigner une partie du corps : par exemple, le thorax est dit « corps de la poitrine » (seezhijn bije), par opposition aux jambes, et le dos " corps derrière » (bögsön bije), par opposition à sa face avant. On peut aussi, par analogie, définir un des versants d'un élément à deux faces -comme une rivière. Le terme bije peut alors prendre le sens de "côté, berge ». Ces usages relèvent d'une conception du corps comme ensemble cohérent, caractérisé par sa matérialité : ses parties le constituent comme elles sont constituées par lui. Dans le langage courant, le corps est donc vivant, sain, matériel et achevé. En totalité, il représente la personne et, dans ses parties, certains de ses aspects.

Mais le corps est aussi le siège d'autres composantes de la personne, qui sont symboliques : ce sont l'âme et la force vitale. La force vitale est générique, elle circule entre les corps. En revanche, l'âme est individuelle et se réincarne. La force vitale est à l'âme ce que la nourriture est au corps (Hamayon 1990 : 443). Sans elle il n'y a pas de vie et l'âme ne peut pas s'incarner dans le corps. L'âme est associée aux os et la force vitale, à la chair et au sang. Les Mongols ont un système de parenté exprimé en termes « d'os et de chair » : les os viennent du père, tandis que le sang et la chair viennent de la mère. L'« unité de vie ", l'« âme ", est ainsi obtenue des ancêtres patrilinéaires de l'enfant, tandis que la constitution du fotus est sous la protection des ancêtres de sa lignée matrilinéaire. Le corps est fait d'os et de chair et son achèvement implique donc, en principe, les ancêtres des deux lignées d'origine de l'enfant.

Quelques attributs de la personne sont considérés comme supports de la force vitale (les cheveux, le souffle, la ceinture, le chapeau, etc.). L'âme est mobile et s'échappe hors $\mathrm{du}$ corps par certains de ses orifices symboliques (aisselles, ombilic, fontanelle). Les motifs de son départ peuvent être réguliers, comme le sommeil, ou occasionnels, comme la peur. Il arrive que l'âme humaine s'échappe à cause des menées d'un esprit qui souhaite l'«enfermer dans un chaudron" pour prendre sa place dans le corps vacant et manger sa force vitale jusqu'à entraîner sa mort. Les motifs de départ de l'âme ou la perte de force vitale comportent donc toujours une part de danger. Toutes les techniques du corps quotidiennes visent d'ailleurs à garantir la présence et la cohésion des composantes symboliques de la personne, moyennant le dressage du corps qui permet que s'incarnent ces dernières. 
Faire un adulte

17 Mauss distingue les techniques de la naissance, de l'obstétrique, les techniques de l'enfance, les techniques de l'adolescence et les techniques de l'âge adulte (1989: 376 ), séparant ainsi les étapes de l'éducation et le moment de la transmission des savoirs acquis. Les techniques du corps varient certes en fonction de l'âge, mais aussi du sexe (1989 : 374).

Dans cet inventaire semblent manquer la gestation et la grossesse. Or chez les Mongols, depuis sa conception, dont l'analyse relève plutôt des techniques de la sexualité ${ }^{2}$, le fœtus est «manipulé » par l'intermédiaire du corps de la femme enceinte. Il s'agit alors de garantir sa constitution. L'accouchement marque l'incarnation initiale de l'âme, mais sa réussite est conditionnée par celle du processus de gestation -c'est-à-dire la grossesse. Il existe parallèlement un rapport étroit entre l'alimentation de la femme enceinte et les représentations de l'accouchement. Il faut donc traiter ensemble la grossesse et l'accouchement. En outre, il ne faut pas limiter les techniques de la naissance à celles de l'obstétrique. En effet, on distingue deux étapes: la naissance biologique puis la naissance sociale.

19 L'enfant traverse ainsi plusieurs étapes depuis sa gestation, sa naissance réelle, puis sociale, la petite enfance, l'enfance et l'adolescence. Il est d'abord accueilli par ses parentes cognatiques, et immédiatement après par ses parents agnatiques. Au fur et à mesure, il est introduit dans des groupes de parenté de plus en plus larges.

L'ensemble du processus de dressage du corps jusqu'à l'âge adulte porte plusieurs noms en mongol : « faire un humain » (xümzhü̈̈le-), « éduquer » (sura-), et " préparer, cuire » (bolovsro-). Les étapes du processus de constitution d'une personne adulte sont caractérisées par différentes techniques du corps dont je n'examinerai que les plus significatives. L'accès à certaines de ces étapes est marqué par un rite de passage dont je n'étudierai pas les détails cérémoniels, mais seulement quelques éléments.

21 Ce choix trouve plusieurs justifications. D'abord, un rite de passage marque la reconnaissance sociale de l'accès à une nouvelle étape du cycle de vie, tandis que les techniques du corps sont les instruments du dressage qui permet cet accès. Les rites de passage sanctionnent aussi l'acquisition et/ou la maîtrise d'une technique du corps particulière. Ensuite, en raison de leur faste, de leur caractère ostentatoire, et parce qu'ils exigent souvent l'intervention d'un spécialiste, les rituels se prêtent plus facilement aux tentatives d'acculturation que les techniques du corps, considérées comme « naturelles».

Les techniques de la gestation

L'arrivée d'un nouveau membre dans la communauté est un événement qui attise la convoitise des humains et provoque l'envie des esprits. Aussi l'annonce d'une grossesse est-elle toujours chose délicate. Elle est retardée au maximum et s'effectue métaphoriquement ${ }^{3}$.

Les Mongols valorisent l'image de la femme travailleuse, et c'est encore plus vrai pour la femme enceinte dont l'activité garantit le bon déroulement de l'accouchement et la santé du fœtus. Il faut certainement voir dans cette prescription d'activités un moyen de faire jouer la "sélection naturelle ». Car si la femme enceinte travaille autant, «l'enfant fragile $»^{4}$ et non viable ne sera pas porté jusqu'à son terme. On préfèrera en effet une fausse couche à la naissance d'un nourrisson mort-né ou à un accouchement difficile, fatiguant et dangereux. En revanche, l'infanticide est réprouvé. L'enfant 
naturel est « venu du ciel » et, si sa mère est (re)mariée, il est éduqué par des parents de celle-ci.

Les techniques du corps de la femme enceinte concernent surtout la maitrise de son alimentation. Tous les "aliments d'importation ", les nourritures d'origine étrangère, comme les céréales ou les épices, lui sont en théorie interdits. Seuls sont autorisés les produits issus de l'économie pastorale nomade: les produits laitiers et la viande d'élevage, à l'exception des viandes peu fraîches ou obtenues à la suite d'un abattage sanglant $^{5}$. Les chairs des animaux dont le temps de gestation est supérieur à celui de la grossesse humaine (chameau ou cerf musqué) pourraient retarder l'accouchement; elles sont également interdites. La viande de prédilection de la femme enceinte est celle du cheval, car le temps de gestation du poulain est de dix mois lunaires, comme le petit d'homme aux yeux des Mongols 6 .

En outre, les usages attribuent à la femme enceinte une part de viande bien particulière : la rotule, caractérisée par ses ligaments. Savoir bien manger la rotule et rendre cet os blanc garantit à la femme enceinte un accouchement facile. Pour les Mongols, l'accouchement provoque une "dislocation des os", censés se détacher au niveau des articulations ${ }^{7}$. La maitrise de la consommation de la rotule témoigne donc de l'acquisition des techniques de consommation de la viande. Elle exprime également la maitrise des articulations du squelette, et par conséquent, celle de la dislocation des os pendant l'accouchement.

Les soins du corps en général, et ceux de la femme enceinte en particulier, sont assez peu réglementés chez les Mongols. Aucune toilette particulière, rituelle ou non, ne lui semble imposée. Néanmoins, pour faciliter symboliquement l'accouchement, les femmes enceintes doivent se gratter et se griffer le ventre avec une patte d'ourse, généralement transmise par leur mère au cours des derniers mois de la grossesse. Cette lacération superficielle est censée garantir un accouchement semblable à la mise bas des ourses, qui "accouchent sans s'en apercevoir", en marchant ou pendant l'hibernation. La démarche comparative peut nous aider à comprendre cette assimilation de la parturition humaine à celle de l'ourse. L'ours est un esprit dispensateur de naissance chez les peuples turcophones, éleveurs et chasseurs de Sibérie, comme les Yakoutes (Dyrenkova 1930 : 63-78; Karunovskij 1927 : 1-40). Il est l'esprit protecteur des enfants chez les Bouriates de Sibérie, peuple mongol d'éleveurs et de chasseurs. C'est encore l'esprit protecteur de la parturition chez les Mongols. Mais, dans la littérature orale de ces derniers, on assimile l'ours à l'écureuil. Or, l'ours comme l'écureuil n'appartiennent pas aux animaux symboliquement importants (" bons a penser ») chez les Mongols où ils ne constituent pas une source de subsistance (il n'est pas chassé), et n'est pas considéré comme un ancêtre d'humain : on ne l'appelle pas par un terme de parenté et on ne lui «demande" pas d'enfant. Ainsi, plus on descend des plaines de Sibérie vers les steppes mongoles, moins l'ours joue un rôle en tant que source de subsistance, et moins sa fonction est importante dans le processus symbolique d'obtention d'enfant. Il est dispensateur d'enfant en Sibérie ou il est un ancêtre d'humain et une source de subsistance tandis qu'il n'est que le protecteur symbolique de l'accouchement des femmes en Mongolie.

Les techniques de la naissance et de l'obstétrique

27 Les techniques de la grossesse visent à "domestiquer " la force vitale du corps de la femme enceinte afin de garantir la constitution du fotus. La grossesse finit avec 
l'accouchement, clos par les relevailles, qui marquent la séparation du nourrisson et de sa mère.

Seuls des femmes et des hommes stériles parents de la parturiente étaient autorisés à participer à l'accouchement, car le sang de la parturition est porteur de fécondité. Son principe peut être agent de souillure pour les hommes, à l'instar du sang menstruel, voire davantage. Chez les Mongols, le principe de résidence est virilocal. Or pour les parents du mari de l'accouchée, et plus spécialement pour son père, la souillure des couches est encore plus dangereuse que pour d'autres personnes. Jusqu'au début du XXe siècle, on montait une yourte plus petite que les autres, spécialement destinée à la parturition ${ }^{8}$, afin de protéger les représentants par excellence du groupe d'affiliation du futur enfant d'une dangereuse impureté. Dans les années 1930-40, la médicalisation de l'accouchement au dispensaire du village s'est imposée sans grande difficulté, car elle garantissait l'éloignement de la parturiente et dispensait de monter cette yourte spécifique.

Dans l'accouchement "traditionnel », la place et la position de l'accouchée avaient également leur importance. Quand elle accouchait sous la yourte, la femme était allongée sur le dos ou à quatre pattes au-dessus du panier réservé au ramassage des bouses (argal) servant de combustible9. La parturiente était couchée sur le sol, parfois sur une litière de crottins (Ar'jaasüren \& Njambuu 1992). Aujourd'hui, à l'hôpital, les Mongoles sont toujours allongées sur le dos. De nos jours encore, il importe que l'enfant «tombe sur le sol» à la naissance. Sous la pression de femmes refusant d'accoucher au premier étage, certains hôpitaux ont ainsi déménagé la salle d'obstétrique au rez-de-chaussée.

L'accouchement sous la yourte était clos par deux rituels : les relevailles, centrées sur l'inhumation du placenta, et la naissance sociale de l'enfant. La sage-femme occupait à cet égard des fonctions essentielles. Elle jouait aussi un rôle pendant la petite enfance, l'enfance et même à l'âge adulte. Elle était réputée pour ses mains « légères, habiles " ${ }^{10}$. Elle pratiquait des massages particuliers sur le ventre de la parturiente en cas de complications des couches. Mais l'habileté manuelle reconnue à la sage-femme renvoie essentiellement à son rôle dans le processus habituel d'une parturition normale.

La sage-femme opérait le passage entre la naissance biologique de l'enfant et sa naissance sociale grâce à une série de manipulations du corps de la mère et du bébé. Après l'avoir mis au monde, la sage-femme lavait le nourrisson pour la première fois, choisissait son nom personnel, et le mettait dans son berceau. Pendant le rituel de la naissance sociale, cette position de "passeuse " était marquée par la part de viande qu'on lui attribuait lors du festin: la "culotte", réservée aux hommes en temps ordinaire. Elle était plus largement signifiée dans le langage alimentaire ${ }^{11}$. La sagefemme était une mère classificatoire de l'enfant, qui la considérait comme sa mère adoptive. Elle assurait le passage d'une maternité biologique à une paternité sociale.

L'accouchement est particulièrement porteur de souillure. Relevant plutôt d'une société de femmes, parentes utérines de l'enfant, il n'a pas fait l'objet d'une tentative de bouddhisation de la part des moines, des hommes particulièrement sensibles, en raison de leur statut, à toute forme de pollution issue du corps. La bouddhisation a donc visé les rituels d'accueil du nourrisson davantage que les relevailles. Le rituel d'inhumation du placenta était ainsi encore pratiqué au début du XXe siècle par les Bouriates (Basaeva 1991, 1993 : 67-91). 
33 Avec la médicalisation de l'accouchement, le placenta n'est plus donné à la mère, mais conservé pour préparer des cosmétiques. Bien que l'inhumation rituelle du placenta soit tombée en désuétude, le morceau de cordon ombilical qui se détache de l'ombilic quelques jours après la naissance est encore employé pour guérir l'herpès du nourrisson. De surcroît, les Mongols attribuent maintenant les vertus régénératrices du lieu d'inhumation du placenta au " souffle vital ${ }^{12}$ du cordon ombilical.

Aujourd'hui, les relevailles sont marquées par la sortie de l'hôpital. De retour au foyer, on procèdera à la naissance sociale de l'enfant. Au XIXe siècle, les Mongols organisaient ensemble ou séparément trois rituels : la première toilette, la dation du nom et la " mise au berceau ». Le souvenir précis de la forme de ces rituels, bien qu'ils fussent bouddhisés, a été conservé en Mongolie jusqu'à aujourd'hui. La médicalisation des couches et la russification ont néanmoins contribué à leur désuétude.

L'influence du bouddhisme se repère grâce à la démarche comparative qui permet de démêler plusieurs contradictions apparentes du rituel de la première toilette. Chez la plupart des Mongols, il est décrit comme un bain : or dans les soins quotidiens, l'enfant n'est jamais immergé, les Mongols estiment même qu'il ne faut pas trop le laver ni le coiffer. Dans les rituels et lors de l'accouchement, le feu, surtout, sert à la purification symbolique. Il est donc étonnant de baigner rituellement l'enfant à la naissance. D'ailleurs, parmi les populations les moins bouddhisées, on ne faisait pas cette toilette. Le nourrisson était au contraire badigeonné de beurre avant d'être langé et mis au berceau. S'il était lavé, ce n'était pas au titre du rituel, mais par des femmes du groupe domestique dans l'intimité de la yourte.

$36 \mathrm{Au}$ XIXe siècle, les populations mongoles " de la forêt » utilisaient encore un berceau sculpté dans une poutre de bois évidée. Il était initialement préparé par un chamane puis transmis entre les germains matrilinéaires (les frères et sœurs de la mère) de l'enfant. La bouddhisation des Mongols et, dans certaines régions, l'extermination des chamanes ont contribué à la disparition des berceaux. De plus, en principe, le berceau ne devait pas être neuf pour ne pas provoquer l'envie des esprits. Les berceaux russes n'ont donc pas remplacé les berceaux traditionnels. Aujourd'hui, les bébés sont simplement langés et posés ficelés sur un lit, «à la russe ». Malgré la tentative de laïcisation du rituel de dation du nom au cours du $\mathrm{XX}^{\mathrm{e}}$ siècle, sa forme actuelle reste très bouddhisée. En outre, les Mongols considèrent que les esprits ne «comprennent pas les langues étrangères » et le choix d'un prénom tibétain permettait de protéger symboliquement l'enfant. Les moines bouddhistes ont assumé une place significative avant la Révolution populaire et la retrouvent aujourd'hui quant au choix et à l'attribution du nom. Les prénoms russes sont également courants.

Les techniques de l'enfance

37 Il y a deux étapes dans l'enfance : la petite enfance, période de « sevrage " jusqu'à trois ans, et l'enfance, qui ouvre sur l'adolescence et la nubilité. Le dressage durant petite enfance vise l'acquisition du langage articulé, des techniques manuelles, d'alimentation et de marche. L'acquisition de la marche ne donne lieu à aucun exercice stéréotypé, et n'est sanctionnée par aucun rituel. La maîtrise des techniques de consommation de la viande va de pair avec l'apparition des dents. Elle ne fait pas l'objet de règles ou d'exercices d'apprentissage particuliers. Les Mongols disposent de beaucoup d'exercices de diction (Chagdarsürüng \& Even 1996 : 289-297), de mémoire ou d'agilité manuelle, destinés aux jeunes enfants (Aubin 1997 : 95-113). 
38 Le rituel de passage de la petite enfance à l'enfance, appelé » fête des trois ans " ${ }^{13}$, correspond à la reconnaissance sociale de deux événements : l'un est physiologique, c'est la fermeture de la fontanelle, et l'autre est cognitif, c'est la maîtrise de la prononciation des mots "pierre» (chuluu) et "cheval» (aduu) ${ }^{14}$. L'ossification de la fontanelle est un signe d'enfermement de l'âme dans le corps. Les Mongols considèrent d'ailleurs la parole comme une marque d'intégrité de la personne, elle témoigne de la présence de l'âme dans un corps vivant. Fixée au corps, l'âme peut ensuite être individuée et l'enfant socialisé. Le processus de sevrage correspond symboliquement à l'humanisation de l'enfant.

39 La maîtrise du langage ouvre l'accès à la consommation de la part de viande réservée aux enfants; auparavant, le petit enfant partage la viande de ses parents. On ne l'appelle plus par son surnom mais par son nom personnel. Il commence à porter des vêtements de fête et n'est plus seulement vêtu de vieux habits. On cesse également de tout lui pardonner : il a désormais des devoirs, des obligations et doit respecter des interdits.

40 Les Mongols organisent encore aujourd'hui la coupe de cheveux de la «fête des trois ans ». Au cours de la petite enfance et avant ce rituel, les cheveux ne sont en principe ni lavés ni coiffés. Actuellement, les Mongols rasent le crâne de l'enfant, en laissant une queue aux garçonnets et deux "cornes" aux fillettes. Cependant, une analyse comparative de plusieurs peuples mongols montre que ce rituel ne concerne le plus souvent que les garçons ${ }^{15}$. De plus, aux niveaux terminologique et symbolique, la " première coupe de cheveux » est assimilée à la castration des poulains, également marquée par la coupe des crins (Humphrey 1970: 70-77). Les rituels de sevrage des enfants d'homme et de castration des poulains ont d'ailleurs lieu au même moment du calendrier saisonnier. La coupe des cheveux/crins est donc une marque corporelle du dressage social, liée à la domestication de la virilité, humaine comme animale.

41 Les techniques de l'enfance concernent le perfectionnement des techniques du corps maîtrisées pour le sevrage: apprendre à manger la part de viande des enfants, maitriser la marche et la course, acquérir une habileté verbale et la "langue de son statut ", connaître les techniques manuelles d'usage. Pendant la socialisation, l'enfant fait l'apprentissage de la division sexuelle des tâches. L'enfance est aussi le moment d'acquérir une nouvelle technique, la monte, ainsi que les différents savoir-faire auxquels elle est indispensable, comme le gardiennage. Les courses de chevaux de la grande fête estivale (Naadam), sont d'ailleurs réservées aux enfants de 6 à 12 ans et, chez certains peuples mongols, aux garçons uniquement.

Les techniques de l'adolescence

42 Avec l'accès à la nubilité, qui s'étend en principe de 11 à 16 ans, une nouvelle étape est franchie : le respect des techniques du corps distinguées en fonction du statut sexuel et générationnel devient obligatoire. La transgression est alors punie par une menace symbolique de stérilité, tandis que pendant l'enfance, elle ne l'était que par des réprimandes. À l'adolescence, tant au niveau biologique et psychologique que social, les rapports au corps changent. Pendant cette période, s'acquièrent les manières d'être et de parler qui caractérisent chacun selon son statut. La future bru et le futur gendre doivent apprendre les interdits linguistiques et gestuels dont la maîtrise caractérisera leur nubilité. Ils finissent l'apprentissage des techniques d'alimentation en apprenant les règles commensales de distribution de la viande. 
43 Le jeune homme commence à expérimenter le maniement de la perche-lasso (uurga), qui lui permettra d'attraper son bétail et qui nécessite une maîtrise parfaite de la monte. La maîtrise complète de la perche-lasso ne s'acquiert qu'à l'âge adulte. En Mongolie, il n'y a pas de rituel initiatique marquant la nubilité. Cependant, la maitrise de trois techniques du corps débouche sur l'éligibilité au mariage. À la fin de la puberté, le jeune garçon doit prouver qu'il est apte à se marier en luttant au moins pendant trois tours contre ses beaux-frères potentiels. Il doit également être en mesure de boire et de chanter trois fois lors des festins. Ces techniques du corps marquent ainsi la sortie de l'adolescence et la nubilité.

Le langage, essentiel à la définition de l'humanité, ne fait pas partie des techniques du corps définies par Mauss. Chez les Mongols, son acquisition donne lieu à un apprentissage important et sa maîtrise conditionne l'obtention d'une épouse. Il conviendrait donc d'inclure la communication verbale à la liste des techniques du corps.

Les techniques de l'âge adulte

Les adultes se divisent en trois catégories: les adultes potentiellement féconds, les adultes sexués ayant fait la preuve de leur fécondité et les anciens sortis du cycle de la reproduction. Pour les femmes, comme pour les hommes, le passage entre la nubilité et l'âge adulte est moins marqué par le mariage que par la naissance du premier enfant.

Mauss distingue chez l'adulte les techniques de repos, d'activité et de mouvement, celles des soins du corps et celles de consommation et de reproduction. Chez les Mongols, dans le processus de constitution d'un adulte, les techniques d'alimentation, de mouvement, les soins des cheveux et l'activité de parole ont un "rendement» important. On pourrait limiter l'analyse à ces techniques que l'efficacité du dressage rend exemplaires ${ }^{16}$.

S'allonger et dormir

47 Les Mongols sont ce que Mauss appelle une société «à natte » et "à oreiller » (1989: 378). Leurs positions de sommeil ne se spécialisent pas en fonction du sexe, mais de l'âge. On distingue le sommeil du bébé, le corps de l'adulte en état de reproduction (activité sexuelle, accouchement) et le sommeil ordinaire des adultes. Le nourrisson emmitouflé dans ses langes dort allongé sur le dos. Après le rituel de la première coupe de cheveux, l'enfant commence à apprendre la position de sommeil caractéristique de l'humain : l'allongement latéral (les adultes s'allongent sur le côté, le dos tourné en direction du centre de la yourte, le visage faisant face aux «murs", xanaa). À l'âge adulte, l'allongement sur le dos est réservé aux techniques de la sexualité et de l'obstétrique.

Le lit est un lieu réservé à l'allongement, sauf lorsqu'il n'y a plus de place pour s'asseoir au centre de la yourte ou pour marquer sa déférence par rapport aux anciens qui y prennent place. Dans la journée, l'allongement est peu apprécié au centre de la yourte. Si une personne fatiguée s'y étend, on lui propose de s'allonger à l'écart, sur un des lits.

$49 \mathrm{Au}$ réveil, le passage entre l'allongement et l'assise autour de la table pour la consommation du petit-déjeuner est marqué par un étirement spécifique. Car s'étirer au réveil permet le retour de l'âme dans le corps après son escapade nocturne, dont le rêve est une manifestation évidente. En dehors du passage du sommeil à la veille, l'étirement est interdit, car l'âme peut s'échapper par les aisselles.

S'asseoir sous la yourte et franchir son seuil 
50 Autour du poêle et de la table, il faut de préférence s'asseoir. L'invité qui pénètre sous la yourte doit s'asseoir et manger. En guise de bienvenue, la femme lui prépare du thé et lui sert de la nourriture, tandis que l'homme lui offre du tabac à priser. L'hôte reste assis à la gauche de l'invité, dans le fond de la yourte, tandis que son épouse au besoin se déplace. Elle doit s'asseoir une fois son travail terminé. Entre membres du groupe domestique également, le centre de la yourte est plutôt un lieu d'assise. On y tolère les déplacements dans le cadre d'une activité, mais ils peuvent paraitre grossiers s'ils ne sont pas motivés. Les manières de s'y asseoir et le lieu de l'assise varient en fonction de l'âge et du sexe. Les anciens s'installent dans le « haut » de la yourte, la partie nommée xojmor, et les enfants, près de la porte. Soulignons que les femmes ont le statut d'enfant classificatoire. En mongol, le terme "femme» (xü̈̈xen) est le singulier du terme « enfant » (xü̈̈xed). Les femmes fécondes ne s'assoient pas au-dessus du poêle, c'est-àdire, dans le xojmor, plutôt réservé aux hommes et surtout aux anciens et aux anciennes. Jeunes femmes et enfants s'assoient donc dans le bas de la yourte. Parallèlement, les formes de l'assise opposent les catégories de personne en fonction du sexe et de l'âge. Les positions de déférence sont ainsi plutôt des assises de femmes ${ }^{17}$.

51 Le campement, autour de la yourte, est un espace qui fait partie de l'habitat, du territoire domestique. Les Mongols évitent de s'y asseoir et surtout de s'y allonger. La sortie sur le campement nécessite de franchir le «seuil » de la yourte, littéralement «[ce] qui se dresse» (bosgo). Il s'agit d'enjamber la petite latte de bois sur laquelle repose la porte. Les techniques d'enjambement du seuil sont très codifiées. Seuls les enfants non sevrés peuvent s'y arrêter ou le traverser à quatre pattes, car ils sont souvent assimilés à du bétail de même âge, poulain ou agneau ${ }^{18}$. Les autres catégories d'humains doivent le franchir debout sur leurs jambes et ne pas s'arrêter. Le passage du seuil doit être effectué de manière parfaite. $Y$ butter donne lieu à une conjuration qui annule les mauvais augures de cette action.

52 En dehors du seuil, l'enjambement est plutôt évité. S'il doit être effectué, sa réalisation est très réglementée. On ne passe jamais au-dessus d'une personne, ou de ses attributs (chapeau, ceinture) et de certains objets liés à des activités viriles ou à l'élevage (harnachement, uurga, etc.). Quand on marche sur le pied de quelqu'un, il faut en principe apaiser le «conflit des jambes » par « l'amitié des mains», en touchant de la main droite la main ou le bras de la personne offensée. À Oulan-Bator, dans la foule qui cherche à monter dans les bus et les tramways, les Mongols évitent de se marcher sur les pieds, et lorsque cela arrive, celui qui a marché sur le pied d'un autre va tout faire pour lui toucher le bras ou la main.

53 Le rapport entre l'entrejambe et les parties génitales est évident. L'interdit d'enjambement est plus général et plus fort pour les femmes que pour les hommes. La " force de captation » de la fécondité associée à l'enjambement ${ }^{19}$ joue également un rôle dans cet interdit, l'enjambement réel fait pendant, au niveau symbolique, à sa faculté de réanimation. Ainsi dans les épopées (Hamayon 1990 : 194), l'enjambement du héros par sa sœur ou sa fiancée permet de le faire revivre. En fait, l'enjambement est réservé au passage entre deux catégories d'espace. Il marque aussi le passage entre deux techniques du corps: les techniques de repos (allongement ou assise) et celles de l'activité et du mouvement.

Les techniques de mouvement

54 À l'âge adulte, les activités imparties à l'homme concernent plutôt la sociabilité élargie, tandis que la femme s'affaire sur le campement. Le travail des femmes impose de 
longues marches autour du campement et celui des hommes, de longues chevauchées sur les pâturages. La marche est donc plutôt une activité féminine et la monte, masculine. La maîtrise de l'uurga nécessitant une certaine dextérité du cavalier est d'ailleurs un savoir-faire exclusif des hommes. L'attitude caractéristique des hommes pendant la marche consiste à croiser les mains dans le dos. Les femmes comme les enfants ne doivent pas l'adopter, sous peine de devenir orphelins. Elle est également déconseillée aux hommes du vivant de leurs parents. Cette manière de marcher est donc particulière aux chefs de famille.

Parallèlement, la course, à pied pratiquée pendant l'enfance est dévalorisée chez l'adulte en dehors d'une activité qui la motiverait. Il est recommandé de ne pas approcher en courant le campement d'une famille, car les chiens n'apprécient pas la course des adultes. Ils ont en revanche l'habitude de celle des enfants. En outre, pour aller vite, un adulte préfèrera souvent son cheval à ses propres jambes. En général, la course est réservée à la partie extérieure de l'espace domestique : le campement pour la course à pied et les alentours du village pour les courses de chevaux. Les principes d'organisation des techniques de mouvement différencient donc la monte, où l'homme doit exceller, la marche surtout nécessaire aux activités féminines, et la course réservée aux enfants.

La classification des techniques de mouvement en fonction des catégories de personne concerne aussi le marquage de la différence entre les laïcs et les spécialistes religieux. Dans les anciens rituels de renouveau du Naadam, la lutte des hommes faisait pendant aux rondes dansées de l'ensemble de la communauté et aux sauts du chamane. Ces activités rituelles étaient effectuées conjointement, au pied des montagnes claniques. Les techniques du corps qui y étaient associées comportent un aspect imitatif. Dans la lutte, l'imitation de cervidés ou de gallinacés constitue une incitation à l'amour adressée au gibier. Dans les danses collectives, les rondes mixtes étaient ponctuées d'onomatopées imitatives de certains gibiers. Ces techniques de non spécialistes nécessitent une grande maitrise du corps. En revanche, les sauts et la gesticulation du chamane pendant la séance ne sont pas chorégraphiés. L'efficacité de la pantomime dépend de la capacité d'improvisation de chaque chamane. Or, en dehors de la séance chamanique, le saut n'est guère apprécié. En outre, au cours de l'initiation, le chamane devait grimper aux arbres. Cette activité lui est en théorie réservée. Il est vrai que dans les steppes mongoles, il y a peu d'arbres. Cependant, dans la taïga septentrionale mongole où vivent les Darxad, les enfants ont peur de monter aux arbres et très peu savent le faire.

À la frontière du campement, il y a toujours un lieu d'attache des chevaux (piquet, longe), et pour pénétrer dans l'espace domestique, on doit mettre pied à terre. Les pâturages sont ainsi plutôt le lieu de la monte. Cela ne signifie pas qu'on n'y marche pas, mais que l'on préfère y aller à cheval. Sur les pâturages, l'éleveur doit souvent mettre pied à terre, mais il est alors libre de le faire à sa guise. En revanche, le franchissement de la frontière entre le campement et les pâturages correspond à des règles bien précises. On doit par exemple attacher les rênes au pommeau de la selle, et le cheval par le licol. L'arrêt à la frontière du campement implique donc la réalisation d'une technique corporelle de " passage » spécifique.

L'analyse des techniques de mouvement révèle comment la pensée classificatoire mongole associe de manière privilégiée une technique du corps et une catégorie d'espace. La yourte est ainsi plutôt le lieu du repos, c'est-à-dire, de l'allongement et de 
l'assise. Le campement est, quant à lui, celui du déplacement à pied et les pâturages, celui du déplacement à cheval. Une technique du corps est donc de préférence associée à un lieu ou au passage d'un lieu à un autre et, réalisée ailleurs, elle peut paraître grossière ou déplacée.

Les techniques de la consommation alimentaire

Parmi les techniques alimentaires, celles de la consommation de la viande ont un rendement important. Il l'est moins en ce qui concerne les laitages ou le thé. À l'âge adulte, les manières de manger ne changent pas. En revanche, la part de viande attribuée à chacun varie en fonction de l'âge, du sexe et du statut social (Hamayon 1975: 99-122). Les techniques de la consommation alimentaire varient néanmoins, encore une fois, au cours du cycle de vie, mais pas à l'âge adulte. Quand le vieillard perd ses dents, il perd aussi l'accès à sa part de viande et mange une nourriture coupée en petits morceaux, comme les jeunes enfants.

En revanche, les manières de boire évoluent au cours de l'âge adulte. La nubilité ouvre l'éligibilité au mariage et l'accès à l'alcool. Ensuite, la consommation d'alcool est autorisée aux adultes, surtout aux hommes. Elle s'accompagne systématiquement de chants ou de bénédictions. Boire de l'alcool est réservé aux adultes et implique la maîtrise du langage. L'ivresse, en revanche, entraîne la perte du langage. Elle est, en théorie, interdite aux adultes mais préconisée pour les vieillards. On prendra soin d'un vieil homme saoul, tandis qu'on évitera la présence d'un jeune homme ivre. On jugera le premier d'un œil bienveillant et le second, avec mépris. Seuls les anciens ayant réalisé leur fécondité, c'est-à-dire, dont les enfants ont eux-mêmes des enfants ou sont en âge d'en avoir, peuvent librement se saouler. L'ivresse est censée provoquer le départ de l'âme par la fontanelle, les aisselles ou le nombril. Autoriser l'ivresse des anciens revient alors à favoriser le départ de l'âme pour préparer la mort.

61 L'accès au statut d'ancien correspond à la sortie réelle ou supposée du cycle de la reproduction. Cela ne marque pas la fin de la sexualité, mais la fin théorique de la fécondité. Une ménopause symbolique touche ainsi les hommes comme les femmes. L'accès à ce statut est souvent associé à un soin du corps particulier. Il concerne les "poils/cheveux» (us) et n'est pas ritualisé. Avec l'accès à la vieillesse, les hommes peuvent se laisser pousser la barbe. A contrario, après la ménopause biologique, certaines femmes se rasent complètements la tête. Elles accèdent de cette façon au statut de «femme consacrée » (chavganc). À l'époque moderne, ce terme désignait les femmes ayant fait leurs vœux bouddhiques, mais aujourd'hui, il correspond simplement au statut de femme ménopausée. L'objectif de cette nouvelle étape du dressage est inversé par rapport à celui du sevrage qui vise à fixer l'âme au corps. Le vieillard perd d'ailleurs progressivement la maîtrise des techniques du corps acquises au cours du sevrage : la parole, le mouvement et la capacité à consommer de la viande.

En guise de conclusion, rappelons que chez les Mongols les techniques du corps à « fort rendement " concernent l'alimentation, le mouvement, les soins des cheveux et le langage. Ces capacités font l'objet d'un appareillage technique important et sont le fruit d'un dressage tout au long de la vie. Une coupe de cheveux rituelle sanctionne l'humanisation achevée des garçons, débouchant sur la domestication de leur fécondité, tandis que le droit de porter la barbe marque la fin théorique de la fécondité des hommes accomplis. L'humanisation des filles était sanctionnée par le perçage de leurs oreilles. En revanche, leur sortie du cycle de la reproduction est marquée par une coupe de cheveux. Ainsi le dressage continue-t-il de la gestation jusqu'à la mort et 
marque-t-il chacune des étapes franchies au cours du cycle de vie. La petite enfance est une période similaire mais inverse de la vieillesse. La première dresse le corps pour y fixer l'âme et permettre son individuation; et la seconde vise à opérer la séparation de l'âme, pour garantir sa circulation, et la mort du corps.

La mise en application concrète de la notion de techniques du corps dans ces sociétés révèle aussi un rapport étroit entre les représentations du corps et celles de l'espace. Il y a, chez les Mongols de tradition pastorale nomade, une connexion privilégiée, mais non absolue, entre une technique du corps et un lieu spécifique. Il existe des techniques corporelles de « passage » entre des lieux, et entre les techniques du corps qui leur sont associées. S'étirer, enjamber, se mettre en selle, et mettre pied à terre, voilà qui marque le franchissement d'une frontière, réelle ou symbolique. Les techniques de mouvement dessinent ainsi un autre axe de lecture synchronique du corps, inscrit dans l'espace. Deux axes de lecture sont donc possibles. Le premier est diachronique et suit un cycle de vie idéal en fonction du sexe, de l'âge et du statut. Le second est synchronique et renvoie aux représentations de l'espace. Étendu à l'ensemble des techniques du corps, l'axe synchronique débouche sur l'analyse des représentations de l'espace. Il est étonnant de constater que les Mongols mettent toujours en valeur la maîtrise de soi et des mouvements. Le mouvement leur permet de diviser et de limiter l'immensité de l'espace qui s'offre à leurs déplacements.

\section{BIBLIOGRAPHIE}

Ar'Jaasüren Ch. \& Njambuu, X.

1992. Mongol jos zanshlyn ix tajlbar tol' [Grand dictionnaire des traditions mongoles]. Ulaanbaatar : Süülenxüü xüüxed.

Aubin, Françoise

1975. » Le statut de l'enfant dans la société mongole », pp. 459-499, in L'enfant : Recueils de la Société J. Bodin; tome 35.

"Sagesse des anciens, sagesse des enfants, dans les steppes mongoles », Enfance: Asie IV : 95-113.

Basaeva, K. D.

1991. Brak i sem »ja u Burjat. Ulan-Ude. Burjatskij : Institut Obshcheswennyx nauk.

1993. » Rites et coutumes concernant les enfants chez les Bouriates de Cisbaikalie », Études

Mongoles et Sibériennes 24 : 67-91.

Chag Darsürüng, Tsevelijn \& Even, M.-Dominique

1996. » Virelangues mongols », Actes de la 37e P.I.A.C. Chantilly, 20-24 juin 1994. Études mongoles

et sibériennes 27 : 289-297.

Dyrenkova, Natalya P.

1930. » Bear worship among Turkish tribes of Siberia », Proc. 23rd int. Cong. of Americanist. New York, 1928, p. 63-78.

Even, M.-Dominique

1998. » L'au-delà dans les représentations religieuses des Mongols », pp.149-196, in P. Servais 
(ed.), La mort et l'Au-delà, Une rencontre de l'Orient et de l'Occident. Louvain-la-Neuve : BruylantAcademia.

Hamayon, Roberte

1970. » Façons de s'asseoir ", Études mongoles $1:$ 135-141.

1975. » L'os distinctif et la chair indifférente », Études mongoles et sibériennes 6 : 99-122.

1990. La chasse à l'âme : esquisse d'une théorie du chamanisme sibérien. Nanterre : Société

d'ethnologie.

Humphrey, Caroline

1970. » Notes on hair-cutting and castration in Mongolian horse herds », Journal of Anglo-

Mongolian Society 2 (1) : 70-77.

Karunovskij, L. E.

1927. » Iz altajskix verovanij i obrjadov, svjazannyx s rebenkom », Sbornik Muzeja antropologii i êtnografii VI :1-40

Mauss, Marcel

1989. » Notion de technique du corps », Sociologie et anthropologie. Paris : Presses Universitaires de France (1ère édition 1950).

\section{NOTES}

1. Ce travail est issu de mes recherches de doctorat sur les techniques du corps (thèse soutenue en 2000 à Paris X-Nanterre), et de leur prolongement post-doctoral sur les règles de l'hospitalité, la consommation d'alcool et les représentations de l'ivresse. J'ai effectué la plupart de mes terrains dans la vallée de Bajanzürx dans la province du Xövsgöl, région de la taïga du nord-ouest de la Mongolie, peuplée à 70\% de Mongols darxad. Les Darxad sont un des peuples minoritaires de Mongolie, dominée par les Xalx. J'ai également réalisé plusieurs terrains chez les Xalx, plutôt localisés dans les steppes centrales du pays, et de courts séjours chez les Bouriates, dans la taïga sibérienne méridionale, au sud du lac Baïkal, et chez les Ordos des steppes arides du nord de la Chine.

2. Nous n'examinerons pas ces techniques-là dans le détail. Soulignons cependant que, dans la sexualité, la femme est assimilée à une jument et l'homme, au cavalier qui la monte, au sens propre comme au figuré. Par cette métaphore, la culture mongole assimile la sexualité au dressage des juments et marque ainsi la maîtrise humaine du processus de reproduction.

3. Il existe quantité de devinettes et de maximes permettant l'annonce d'une grossesse à son époux, à ses beaux-parents, et à sa famille d'origine ou à son groupe de filiation.

4. En Mongolie, la mortalité infantile était importante jusqu'au milieu du XXe siècle. Aujourd'hui encore, l'enfant fragile est désigné par l'expression " enfant qui ne se fixe pas » (togtoxgüj xü̈̈xed). Les procédures pour le protéger sont multiples : adoption, changement de nom personnel, inversion du sexe par le vêtement, etc. (Aubin 1975 : 459-499).

5. Les Mongols pratiquent un abattage sans effusion de sang. Ils sectionnent l'aorte en glissant la main par une incision effectuée au niveau du thorax. L'animal meurt d'une hémorragie interne. Le sang recueilli dans l'abdomen est utilisé pour faire des boudins. 6. L'expression « naître après dix mois révolus » désigne la naissance sans complication d'un bébé bien constitué. 
7. La distribution de la viande n'est jamais aléatoire. À chaque catégorie de personne correspond une part précise. En contexte de virilocalité, le bétail consommé provient idéalement du troupeau du beau-père ou du mari. On donne la tête au groupe du mari et la poitrine à celui de l'épouse ou de la bru. Les pattes sont une part d'enfant, etc. 8. Les éleveurs possèdent souvent, en plus de la yourte d'habitation, une autre yourte, plus petite, qui sert en différentes occasions : comme yourte de transhumance (otor ger) en cas d'intempéries imposant le déplacement d'une partie du troupeau sur d'autres pâturages, comme yourte d'isolement lors de maladie contagieuse ou de souillure symbolique, ou simplement comme débarras, pour le rangement, la cuisine, etc.

9. C'est l'activité féminine par excellence et le contact avec ce panier à argal est interdit aux hommes.

10. Tout comme le castrateur de chevaux. Ces images sont récurrentes dans la pensée populaire mongole qui exprime, par là, la maîtrise humaine du processus de reproduction de l'homme comme de l'animal.

11. Chez les Mongols xalx, les parts de viande utilisées pour préparer l'eau du bain de l'enfant servent aussi à la préparation d'un bouillon, interdit de consommation pour l'accouchée, mais prescrit à la sage-femme. Au sein d'autres ethnies mongoles, l'eau du bain est parfois préparée à partir de thé au lait. Sa consommation est alors interdite à la mère et préconisée pour la sage-femme.

12. Dans le traitement de la naissance des jumeaux, le cordon ombilical est défini comme un support de souffle et un siège de force vitale. Chez les peuples mongols, les jumeaux sont censés avoir deux âmes, mais un seul souffle. Ils sont « fragiles » et représentent un excès de force vitale qu'il faut cacher. On effectuait jusqu'à une période récente un rituel de "séparation du souffle », c'est-à-dire, une manipulation des cordons ombilicaux des jumeaux. Après ce rituel, les jumeaux étaient assimilés à de simples frères et sœurs.

13. Ce rituel est organisé une année impaire d'âge pour les garçons, trois ou cinq ans, et une année paire pour les filles, quatre ou six ans. La comparaison de ce rituel organisé par plusieurs peuples mongols révèle que ces termes d'âge sont rajoutés a posteriori pour désigner la période de maîtrise du langage.

14. Soulignons que ces deux mots ne sont pas particulièrement significatifs de la phonologie du mongol, qui oppose généralement deux principes d'harmonie vocalique : masculin/féminin ou antérieur/postérieur. Aduu et chuluu appartiennent à la même harmonie vocalique : masculin-antérieur. En revanche, ils sont culturellement significatifs de deux images chères aux Mongols : le cavalier et le terroir.

15. Cette première manipulation des cheveux de l'enfant ouvre un cycle de marquage du statut social par la coiffure. Les Xal'mg, Mongols occidentaux (Ojrat), font d'autres coupes de cheveux, mais pour les garçons uniquement. Ils marquent ainsi l'entrée dans l'adolescence et la maîtrise du maniement de la perche-lasso (uurga). Par ailleurs, dans l'idéal mongol, les cheveux des femmes représentent leur fécondité. On ne les coupe jamais en dehors de ce rituel. Plusieurs éléments invitent à penser que les Mongols de Mongolie auraient organisé, en parallèle à la coupe de cheveux des garçons, le perçage des oreilles des filles, rituel qui n'existe plus.

16. Je n'étudierai pas ici les soins du corps caractérisant l'âge adulte, ni les techniques de la reproduction, car elles sont complexes et demanderaient une étude spécifique. 17. S'agenouiller est l'assise préférée des femmes. Or cette position est aussi l'assise de prière devant une divinité bouddhique. En revanche, l'assise en tailleur est interdite aux femmes et aux enfants. C'est l'assise de prédilection des Bouddhas dans les icônes 
qui leur sont consacrées, des lamas, et des hommes au centre de la yourte (Hamayon 1970 : 135-141).

18. Les seuls animaux à franchir le seuil de la yourte sont d'ailleurs les chevreaux et les agneaux que l'on protège à leur naissance des prédateurs et du froid des nuits printanières.

19. Par exemple, la femme enceinte ne doit pas enjamber un arbre, « comme une ourse ", animal dont elle cherche pourtant la protection symbolique en se lacérant le ventre avec une de ses pattes. Si elle en cherche la protection, elle ne souhaite pas être confondue avec cet animal.

\section{RÉSUMÉS}

Cet article propose une mise en application aux peuples mongols de la notion de «technique du corps » telle qu'elle a été définie par Marcel Mauss. Dans ces sociétés, le «dressage humain » est continu tout au long de la vie. La gestation fait partie intégrante des «techniques de la naissance et de l'obstétrique » et il existe un dressage du vieillard, en quelque sorte inversé par rapport à celui de l'enfant. En outre, les Mongols de tradition pastorale nomade organisent l'espace en fonction de la motricité du corps. Il existe donc deux axes de lecture des techniques du corps mongoles. Un axe diachronique qui concerne le dressage et un autre, synchronique, qui renvoie à l'organisation de l'espace. Sur ces deux axes, se répartissent des techniques de passage.

Mongol corporal technics : using Mauss's concept. This articles proposes to apply Marcel Mauss's corporal techniques concept to Mongol populations. In these societies « human raising » is continuous through the entire life of the individual, from gestation to old age. For old men "raising" is reversed compared to childs' one. Besides, for Mongol people of nomadic and pastoral tradition, space is organized according to body movements. There are thus two ways of reading mongol corporal technics : one is diachronic, concerning raising, the other is synchronic, which deals with space organization; both of them including « passing through » technics

Las técnicas del cuerpo mongoles. Una aplicación de la noción de Mauss. El artículo aplica a los Mongoles la noción de técnica del cuerpo tal como fue definida por Marcel Mauss. En estas sociedades, la «crianza humana " es contínua a lo largo de la vida. La gestación es parte integrante de las « técnicas del naciemiento y de la obstétrica ", y existe algo como una " crianza del anciano» que parece invertida si se compara con la del niño. Además, los Mongoles cuya tradición sigue siendo pastoral organizan el espacio en funcción de la motricidad del cuerpo. Así pues, existen dos ejes de lectura de las técnicas mongoles del cuerpo: un eje diacrónico que respecta à la crianza, y otro, en el marco de la organización del espacio. Unas técnicas de paso marcan ambos ejes.

\section{INDEX}

Mots-clés : Mauss (M.), alimentation, corps, enfance, gestation, Mongols, naissance, rites de passage, espace 
AUTEUR

GAËLLE LACAZE

Centre d'études mongoles et sibériennes, Paris EPHE-Section des sciences religieuses. 